\title{
QUALIDADE DE AMEIXAS CV. REUBENNEL APÓS TRATAMENTO COM PRODUTOS ALTERNATIVOS, REFRIGERAÇÃO E CLIMATIZAÇÃO ${ }^{1}$
}

\author{
ELIANE APARECIDA BENATO², MARIA FERNANDA PENTEADO MORETZSOHN DE CASTRO ${ }^{3}$, \\ JOSÉ MARIA MONTEIRO SIGRIST ${ }^{3}$,VALÉRIA DELGADO DE ALMEIDA ANJOS ${ }^{3}$, \\ SILVIA REGINA TOLEDO VALENTINI ${ }^{4}$
}

RESUMO - O objetivo deste trabalho foi avaliar a aplicação de produtos alternativos e de refrigeração, com posterior aplicação de etileno, sobre a qualidade de ameixas 'Reubennel', a fim de prolongar o período de comercialização. Os produtos aplicados logo após a colheita foram: biomassa cítrica (Ecolife $($ ) - $150 \mathrm{~mL}$ (p.c.) $100 \mathrm{~L}^{-1}$ por $30 \mathrm{~s}$; vapor de etanol $-2 \mathrm{~mL} \mathrm{~kg}^{-1}$ por $2 \mathrm{~h}\left(20^{\circ} \mathrm{C} / 80 \% \mathrm{UR}\right)$, e cloreto de cálcio $\left(\mathrm{CaCl}_{2}\right)-1 \%$ (p/v) por 10 min. Em seguida, os frutos foram armazenados a $0^{\circ} \mathrm{C} / 95 \%$ UR. Após 17 e 33 dias, três caixas de cada tratamento, com 40 frutos cada, foram removidas da câmara de refrigeração, sendo uma das caixas segregada para análises, enquanto as outras duas foram transferidas para $20^{\circ} \mathrm{C} / 85 \% \mathrm{UR}$ por mais 4 dias. Destas caixas, uma de cada tratamento foi submetida à climatização, através da aplicação de etileno $(100 \mathrm{uL}$ $\mathrm{L}^{-1}, 72$ h) em minicâmara (200 L). Procedeu-se à avaliação da qualidade dos frutos ao zero; 17; 17+4; 33 e $33+4$ dias, através de análises físico-químicas, microbiológicas e sensoriais. Os resultados foram analisados estatisticamente, em delineamento experimental inteiramente casualizado para os produtos alternativos e em fatorial $(4 \times 2)$ para a combinação com a climatização. Concluiu-se que o tratamento pós-colheita das ameixas com vapor de etanol reduz a população microbiana, bem como proporciona incremento dos sólidos solúveis. As ameixas 'Reubennel' podem ser armazenadas por até 33 dias sob refrigeração $\left(0^{\circ} \mathrm{C} / 95 \% \mathrm{UR}\right)$, seguindo-se a climatização com etileno e, imediatamente, comercializadas.

Termos para indexação: Prunus salicina, etileno, etanol, cloreto de cálcio, biomassa cítrica.

\section{PLUMS QUALITY CV. REUBENNEL AFTER TREATMENT WITH ALTERNATIVE PRODUCTS, COLD STORAGE AND CLIMATIZATION}

\begin{abstract}
The purpose of this research was to evaluate the application of alternative products and refrigeration, with subsequent exposure to exogenous ethylene on quality of plums 'Reubennel', in order to prolong the marketing period. Products applied soon after harvest were: citric biomass (Ecolife $\left.{ }^{\circledR}\right)$ - $150 \mathrm{ml}$ (pc) $100 \mathrm{~L}^{-1}$ for $30 \mathrm{~s}$; ethanol vapor $-2 \mathrm{ml} \mathrm{kg}{ }^{-1}$, per $2 \mathrm{~h}\left(20^{\circ} \mathrm{C} / 80 \% \mathrm{RH}\right)$ and , calcium chloride $\left(\mathrm{CaCl}_{2}\right)-1 \%$ $(\mathrm{w} / \mathrm{v})$ for $10 \mathrm{~min}$. Then, the fruits were stored at $0^{\circ} \mathrm{C} / 95 \%$ RH. After 17 to 33 days, three boxes per treatment with 40 fruits each were removed from the cooling chamber, one of the boxes segregated for analysis, while the other two were transferred to $20^{\circ} \mathrm{C} / 85 \% \mathrm{RH}$ for 4 more days. Of these boxes, one for each treatment was subjected to climatization through the application of ethylene $\left(100 \mathrm{uL}^{-1}, 72 \mathrm{~h}\right)$ in a mini-camera $(200$ L). We proceeded to evaluate the quality of the fruit at zero, 17, 17+4, 33, and 33+4 days, through physicalchemical, microbiological and sensory analysis. The results were analyzed statistically in a completely randomized design for alternative products and factorial $(4 \times 2)$ for the combination with the climatization. It was concluded that post-harvest treatment of plums with ethanol vapor reduces the microbial population as well as providing increased soluble solids. The 'Reubennel' plums can be stored for up to 33 days under refrigeration $\left(0^{\circ} \mathrm{C} / 95 \% \mathrm{RH}\right)$, followed by climatization with ethylene and immediately commercialized. Index terms: Prunus salicina, ethylene, ethanol vapor, calcium chloride, citric biomass.
\end{abstract}

\footnotetext{
${ }^{1}$ (Trabalho 309-13). Recebido em: 26-08-2013. Aceito para publicação em; 06-02-2015.

${ }^{2}$ Dra., Pesq. Cientifica, Instituto Biológico. E-mail: ebenato@biologico.sp.gov.br.

3Pesq. Cientifico, Instituto de Tecnologia de Alimentos. E-mails: fernanda@ital.sp.gov.br; jmms@ital.sp.gov.br; vanjos@ital.sp.gov.br ${ }^{4}$ Pesq. Cientifica, Instituto Agronômico. E-mail: valentini@iac.sp.gov.br.
} 


\section{INTRODUÇÃO}

Dentre as cultivares de ameixa introduzidas e plantadas em várias regiões do Brasil, destaca-se a 'Reubennel' (Prunus salicina Lindl.). Os frutos são de tamanho médio a grande, de forma redonda cônica e com a epiderme de coloração amarelo-esverdeada evoluindo para vermelho; a polpa é amarela, doce e levemente ácida (NASCIMENTO et al., 1993). Devido à curta safra no Brasil, entre novembro e janeiro, ocorre o favorecimento da entrada de ameixas importadas para a complementação de oferta sazonal. Assim, o armazenamento dessa fruta é uma alternativa viável para estender o período de oferta do produto nacional (BRACKMANN et al., 2001). Por ser um fruto de alta perecibilidade, devido a sua elevada taxa metabólica durante a maturação, a principal forma adotada para reduzir o metabolismo e aumentar o tempo de conservação dos frutos é o resfriamento (BRACKMANN et al., 2005; MALGARIM et al., 2007).

A cultivar Reubennel pode ser conservada por cerca de quatro semanas a $0^{\circ} \mathrm{C} / 90-95 \% \mathrm{UR}$, sendo resistente ao escurecimento interno (internal browning ou internal breakdown), apresentando mudanças pouco pronunciadas nas características físico-químicas durante o armazenamento (KLUGE et al., 1995; MALGARIM et al., 2007). A maioria das cultivares de ameixas mostra-se suscetível ao dano pelo frio (chilling injury) quando armazenada a $5^{\circ} \mathrm{C}$. Além disso, após o armazenamento refrigerado prolongado, pode-se observar significativa perda de massa e incidência de podridões, limitando o período de comercialização. As principais doenças que ocorrem após a colheita das ameixas podem ser causadas por Monilinia fructicola, Botrytis cinerea, Rhizopus stolonifer, Cladosporium sp., entre outros patógenos (CRISOSTO; KADER, 2000; GONÇALVES, 2005).

Estudos mostram que podem ser adotados tratamentos complementares à refrigeração visando à conservação da qualidade dos frutos, como o uso de cloreto de cálcio (GONÇALVES, 2005), biomassa cítrica (ABREU et al., 2008), vapor de etanol (RITENOUR et al., 1997; LICHTER et al., 2006), atmosfera modificada (MALGARIM et al., 2005; STEFFENS et al., 2009) e 1-metilciclopropeno (1-MCP) (ARGENTA et al, 2003; ERKAN; ESKI, 2012). A ameixa é um fruto que apresenta comportamento respiratório do tipo climatérico, com taxa de respiração que varia de 8-12 $\mathrm{mL} \mathrm{CO}_{2}$ $\mathrm{kg}^{-1} \mathrm{~h}^{-1}$ a $20^{\circ} \mathrm{C}$, e a produção de etileno varia numa taxa de $0,1-200 \mu \mathrm{L} \mathrm{C}_{2} \mathrm{H}_{4} \mathrm{~kg}^{-1} \mathrm{~h}^{-1}$ a $20^{\circ} \mathrm{C}$. Para acelerar e/ou uniformizar o amadurecimento dos frutos, pode-se realizar a aplicação exógena do etileno (CRISOSTO; KADER, 2000; FIORAVANÇO et al., 2007; ERKAN; ESKI, 2012), contudo, há correlação positiva entre a produção de etileno e a suscetibilidade de ameixas ao distúrbio pelo frio (CANDAN et al., 2011).

O objetivo deste trabalho foi avaliar a aplicação de produtos alternativos e de refrigeração, com posterior aplicação de etileno, sobre a qualidade de ameixas 'Reubennel', a fim de prolongar o período de comercialização.

\section{MATERIAL E MÉTODOS}

Ameixas 'Reubennel', procedentes da região produtora de Turvolândia-MG, foram transportadas ao ITAL, em Campinas-SP. Inicialmente, o lote de frutos foi selecionado e uniformizado pelo estádio de maturação "meio maduro", ou seja, frutos firmes e com cor de casca amarelo-esverdeada, com traços de vermelho.

Os frutos foram submetidos aos seguintes tratamentos com produtos alternativos: 1) testemunha; 2) Ecolife $\AA$ (biomassa cítrica), 150 $\mathrm{mL}$ (p.c.) $100 \mathrm{~L}^{-1}$, em imersão por $30 \mathrm{~s} ; 3$ ) etanol, 2 $\mathrm{mL} \mathrm{kg}{ }^{-1}$, em vaporização por $\left.2 \mathrm{~h}\left(20^{\circ} \mathrm{C} / 80 \% \mathrm{UR}\right) ; 4\right)$ cloreto de cálcio $\left(\mathrm{CaCl}_{2}\right), 1 \%(\mathrm{p} / \mathrm{v})$, em imersão por 10 min. Cada tratamento contou com 240 ameixas, distribuídas em seis caixas de papelão (40 frutos/ caixa) e, no mesmo dia, foram armazenadas a $0^{\circ} \mathrm{C} \pm 0,5^{\circ} \mathrm{C} / 90-95 \% \mathrm{UR}$.

Após 17 e 33 dias, três caixas de frutos de cada tratamento foram removidas da câmara de refrigeração. Uma caixa foi tomada como amostra para análises, enquanto as outras duas foram transferidas para $20^{\circ} \mathrm{C} \pm 1^{\circ} \mathrm{C} / 85-90 \% \mathrm{UR}$ por mais 4 dias, sendo que uma das caixas de cada tratamento sofreu climatização, através da aplicação da mistura gasosa Etil-5 (100 uL L-1 etileno, por $72 \mathrm{~h}$ ), em minicâmara $(200 \mathrm{~L})$, com intervalos de $24 \mathrm{~h}$ (três aplicações consecutivas a zero, 24 e 48 h, com prévia exaustão).

Ao zero; $17 ; 17+4 ; 33$ e $33+4$ dias, foi tomada uma caixa de cada tratamento, com 40 frutos, para realização de análises físico-químicas, microbiológicas e sensoriais.

Tomaram-se 10 frutos por tratamento para as análises físico-químicas. Para análise de cor de casca, empregou-se colorímetro Minolta, Chroma meter CR-300, sistema $L^{*} a^{*} b^{*}$, tomando-se a leitura da cor em dois pontos opostos na região equatorial de cada fruto. Para a determinação da firmeza da polpa (N), utilizou-se de Texturômetro TA-xT2i, com probe $6 \mathrm{~mm}$ de diâmetro, profundidade de penetração 
$6 \mathrm{~mm}$, velocidade $2 \mathrm{~mm} \mathrm{~s}^{-1}$, com leitura em dois pontos opostos da região equatorial do fruto, após remoção da casca na região da leitura. O teor de sólidos solúveis ( $\left.{ }^{\circ} \mathrm{Brix}\right)$, de cada amostra composta pelo suco de dois frutos, foi medido em refratômetro digital Schmidt + Haensch SR400. Para a acidez titulável, empregou-se $\mathrm{NaOH}(0,1 \mathrm{~N})$ para titulação até atingir $\mathrm{pH} 8,1$, com o resultado expresso em ácido cítrico $\left(\mathrm{g} 100 \mathrm{~g}^{-1}\right)$. Avaliou-se a incidência (\%) de podridões nos 40 frutos de cada tratamento, através da presença ou ausência de sintomas, e procedeu-se também à identificação dos principais patógenos. A incidência (\%) de escurecimento de polpa foi avaliada visualmente, pela presença ou ausência, em 20 frutos por tratamento.

Para as análises microbiológicas, foram utilizados $25 \mathrm{~g}$ retirados aleatoriamente de cada amostra de tratamento composta por dois frutos, com quatro repetições. Tomou-se por base a resolução RDC n.12, de 02-01-2001 (BRASIL, 2001); contudo, devido à ameixa apresentar alta acidez, adicionalmente, foram realizadas as análises de contagem total de microrganismos aeróbios mesófilos e de contagem de bolores e leveduras. As análises de coliformes totais e Escherichia coli foram realizadas utilizando-se de Petrifilms da 3M Company. A análise de Salmonella spp. foi efetuada somente na amostra inicial do lote (DOWNES; ITO, 2001).

Para a análise sensorial das ameixas, aplicouse o teste da escala hedônica de nove pontos para qualidade global, ou seja, 1= desgostei muitíssimo; $2=$ desgostei muito; $3=$ desgostei regularmente; $4=$ desgostei ligeiramente; $5=$ indiferente; $6=$ gostei ligeiramente; $7=$ gostei regularmente; $8=$ gostei muito, e 9= gostei muitíssimo (MORAES, 1988). Contou-se com 7-10 provadores, previamente instruídos para realizar a análise sensorial, que também puderam comentar sobre os atributos de que "mais gostou" e "menos gostou" quanto à textura e sabor (doce, ácido e amargo) e a intenção de compra ( $\operatorname{sim}$ ou não).

As médias dos resultados foram submetidas à análise de variância e comparadas pelo teste de Tukey $(\mathrm{p} \leq 0,05)$. O delineamento experimental foi o inteiramente casualizado, com 4 tratamentos de 10 a 40 repetições em função da análise, aos 17 e 33 dias de armazenamento, enquanto, aos $17+4$ e $33+4$ dias, o delineamento foi um fatorial ( 4 x 2), com 4 tratamentos de produtos alternativos e 2 de etileno. Adotou-se o programa estatístico Estat versão 2.0.

\section{RESULTADOS E DISCUSSÃO}

A caracterização inicial da matéria-prima mostrou que a cor da casca estava com $\mathrm{L}^{*}=49,44, \mathrm{a}^{*}$ $=6,98$ e $^{*}=16,76$; a firmeza da polpa com 8,07 N; o teor de sólidos solúveis com $11,5^{\circ}$ Brix, e a acidez titulável com 1,20 ( $\mathrm{g}$ ácido cítrico $100 \mathrm{~g}^{-1}$ ).

De acordo com os resultados obtidos, posteriormente, pôde-se constatar que as ameixas escureceram $\left(\mathrm{L}^{*}=\right.$ luminosidade) com o tempo de armazenamento, ligeiramente, quando sob refrigeração $\left(0^{\circ} \mathrm{C}\right)$ e, mais acentuadamente, quando transferidas para condições ambiente $\left(20^{\circ} \mathrm{C}\right)$. Frutos climatizados com etileno escureceram, significativamente, quando comparados com os não tratados. Quanto à mudança de coloração da casca $\left(a^{*}, b^{*}\right)$, verificou-se que as ameixas estavam, inicialmente, em estádio amarelo-esverdeado com traços de vermelho. Sob refrigeração, os frutos evoluíram para coloração vermelho-amarelada com decréscimo de verde. Quando transferidos para condições-ambiente $\left(20^{\circ} \mathrm{C}\right)$, aos $17+4$ e $33+4$ dias, notou-se que a aplicação de etileno promoveu, significativamente, a perda do amarelo e a intensificação do vermelho, independentemente dos produtos alternativos (Tabelas 1 e 2) .

Os resultados observados neste trabalho confirmam que o resfriamento de ameixas cv. Reubennel, por aproximadamente 30 dias, é um dos principais processos para se reduzir o metabolismo dos frutos, conservar a qualidade e inibir o desenvolvimento de podridões (KLUGE et al., 1995; MALGARIM et al., 2005). O aumento da intensidade da cor típica desta cultivar ocorre simultaneamente ao pico climatérico e ao incremento da produção de etileno, endógeno ou exógeno, sendo pouco influenciado por tratamentos complementares (FIORAVANÇO et al., 2007; MALGARIM et al., 2007; KLUGE et al., 2008).

Quanto à firmeza da polpa (Tabelas 3 e 4), em geral, as ameixas sofreram amaciamento da polpa durante o armazenamento, mais acentuadamente, sob condições ambiente $(8,07 \mathrm{~N}$ para 5,48 N). Notou-se que os frutos da testemunha permaneceram mais firmes, de modo significativo, quando comparados com os dos outros tratamentos. Porém, o tratamento com etileno promoveu uma redução altamente significativa $(\mathrm{p} \leq 1 \%)$ na firmeza das ameixas $(1,1$ $\mathrm{N}$ ), independentemente dos produtos alternativos aplicados.

A textura da ameixa é um dos parâmetros de qualidade mais importantes, confirmado pela preferência dos provadores na avaliação sensorial. Com o avanço do amadurecimento, os frutos vão- 
se tornando mais macios devido à diminuição da turgescência e à hidrólise das substâncias pécticas que compõem a parede celular (MALGARIM et al., 2007). Há divergências na literatura quanto ao efeito de produtos alternativos sobre o amadurecimento de frutos; como exemplo, foi verificado que vapor de etanol reduziu a atividade de enzimas petcnolíticas em goiabas e manteve a firmeza da polpa por mais tempo; no entanto, acelerou o amadurecimento de bananas (SIDDIQUI et al., 2005). O etileno exógeno acelera o processo de amadurecimento e senescência (KLUGE et al., 2008). Argenta et al. (2003) citam que a taxa de firmeza de $9 \mathrm{~N}$ em ameixas já é considerada própria para consumo.

O teor de sólidos solúveis (Tabelas 3 e 4), praticamente, não variou durante o armazenamento dos frutos, ficando em aproximadamente $11,5^{\circ} \mathrm{Brix}$, semelhante aos resultados de Malgarim et al. (2007). Entretanto, observou-se que o tratamento com etanol favoreceu um acréscimo altamente significativo de sólidos solúveis. Tem sido demonstrado que o etanol e o acetaldeído podem induzir respostas fisiológicas e bioquímicas nos frutos pós-colheita, incrementando o teor de açúcares, reduzindo a acidez e aumentando a preferência sensorial (PESIS, 2005); contudo, Ritenour et al. (1997) não observaram efeito da exposição de ameixas ao vapor de etanol $(<6 \mathrm{~mL}$ $\left.\mathrm{kg}^{-1}\right)$ sobre a firmeza e os sólidos solúveis.

Durante o armazenamento, houve uma tendência de ligeiro decréscimo da acidez nas ameixas de todos os tratamentos (1,2 para $0,9 \mathrm{~g}$ $100 \mathrm{~g}^{-1}$ ac. cítrico), sendo que a aplicação de etileno acarretou redução da acidez de modo significativo (Tabelas 3 e 4). A redução da acidez nas ameixas é consequência do metabolismo contínuo e da respiração, que usa ácidos orgânicos como substrato, estimulada pela exposição ao etileno exógeno (ARGENTA et al., 2003; MALGARIM et al., 2005).

Constatou-se escurecimento de polpa nas ameixas somente após 33+4 dias de armazenamento, em cerca de $35 \%$ dos frutos não climatizados, enquanto os frutos tratados com etileno apresentaram menor porcentagem ( $5 \%$ ) deste distúrbio fisiológico. A exposição prolongada de ameixas a baixas temperaturas pode ocasionar a degenerescência da polpa, entretanto autores relatam que a porcentagem deste distúrbio em ameixas é maior nas cultivares que apresentam alta taxa de produção de etileno, podendo ser reduzida pelo tratamento com 1-MCP(CANDAN et al., 2011; ERKAN; ESKI, 2012). Além disso, a cultivar Reubennel apresenta baixa suscetibilidade a este distúrbio (BRACKMANN et al., 2005; MALGARIM et al., 2007). Dados mais similares aos resultados deste trabalho foram observados em pêssegos, por Palou et al. (2003), que apresentaram incidência de 13\% de internal breakdown após 14 dias de armazenamento refrigerado sob atmosfera de etileno exógeno, contra $60 \%$ do tratamentocontrole. A incidência de podridão nos frutos foi muito esporádica, não permitindo obter resultados conclusivos sobre a eficiência de controle dos produtos alternativos. Os patógenos identificados como causadores das podridões nos frutos foram: leveduras, Cladosporium, Botrytis, Colletotrichum e Monilinia. Malgarim et al. (2007) e Kluge et al. (2008) também verificaram um percentual baixo (5\%) de podridões em ameixas pós-colheita.

As análises microbiológicas indicaram, de modo geral, que os tratamentos com vapor de etanol e cloreto de cálcio promoveram uma redução significativa na população microbiana, composta predominantemente de leveduras. As análises nas amostras de ameixas recém-colhidas mostraram ausência de Salmonella spp. e de coliformes termotolerantes, indicando que as ameixas estavam de acordo com os padrões microbiológicos estabelecidos pela Resolução RDC-12 da ANVISA (BRASIL, 2001).

A efetividade do uso do vapor de etanol no controle de bolores em frutas durante o armazenamento é relatada por Siddiqui et al. (2005) e Lichter et al. (2006), que atribuem a eficácia do etanol à conversão pela baga,de álcool deidrogenase a acetaldeído, o qual é muito tóxico aos microrganismos. Encontram-se relatos da eficiência do $\mathrm{CaCl}_{2}$ no controle de doenças pós-colheita, devido a seu efeito em retardar o amadurecimento dos frutos, consequentemente, dificultando o desenvolvimento de microrganismos (GONÇALVES, 2005). Segundo Abreu et al. (2008), biomassa cítrica mostrou-se eficiente no controle de Monilinia fructicola em pêssegos pós-colheita, porém não controlou a podridão de Rhizopus stolonifer.

Os resultados da análise sensorial mostraram que os provadores avaliaram a amostra inicial, como "desgostei regularmente" a "indiferente" (nota $4,7)$, apontando terem gostado da textura $(8,0 \mathrm{~N})$ e não gostaram da acidez (1,2\%). Aos 17 e aos 33 dias de armazenamento refrigerado, os provadores mostraram-se praticamente "indiferentes" aos tratamentos com produtos alternativos, apontando gostarem da cor, textura e sabor adocicado $\left(11,7^{\circ} \mathrm{Brix}\right)$, porém não gostarem da acidez. Aos $17+4$ e $33+4$ dias de armazenamento, a preferência foi maior pelos frutos tratados com etanol (não climatizados), significativamente diferente da testemunha, sendo que as características mais apreciadas foram novamente cor, textura e sabor doce, e as não 
apreciadas foram a acidez e um ligeiro sabor amargo. Nos frutos tratados com etileno, notou-se maior preferência pela testemunha, devido à cor, suculência e sabor doce, enquanto as características não apreciadas nos frutos, em geral, foram a textura muito mole $(1,1 \mathrm{~N})$, o ligeiro sabor amargo e sobremaduros (dados não apresentados). Malgarim et al. (2007) constataram a plena aceitação dos provadores pelas ameixas armazenadas a $0^{\circ} \mathrm{C}$ por 30 dias, e referem que a presença de ligeiro sabor amargo nos frutos maduros deve estar relacionada ao aumento do teor de compostos fenólicos, vinculado à coloração, ao escurecimento e ao sabor desenvolvidos com o amadurecimento e a senescência.

TABELA 1- Coloração da casca ( $\mathrm{L}^{*}=$ luminosidade; $+\mathrm{a}^{*}=$ vermelho; $-\mathrm{a} *=$ verde; $+\mathrm{b} *=$ amarelo; $-\mathrm{b}^{*}=$ azul) de ameixas cv. Reubennel, submetidas a tratamentos pós-colheita, armazenadas a $0^{\circ} \mathrm{C}$ por 17 e 33 dias.

\begin{tabular}{lcccccc}
\hline \multirow{2}{*}{ Tratamentos } & \multicolumn{2}{c}{$\mathrm{L}^{*}$} & \multicolumn{2}{c}{$\mathrm{a}^{*}$} & \multicolumn{2}{c}{$\mathrm{b}^{*}$} \\
\cline { 2 - 7 } & 17 dias & 33 dias & 17 dias & 33 dias & 17 dias & 33 dias \\
\hline 1)testemunha & $46,66 \mathrm{a}^{(1)}$ & $43,43 \mathrm{a}$ & $7,61 \mathrm{a}$ & $13,75 \mathrm{ab}$ & $21,27 \mathrm{a}$ & $21,96 \mathrm{a}$ \\
2)ECOLIFE® & $47,20 \mathrm{a}$ & $41,92 \mathrm{a}$ & $7,84 \mathrm{a}$ & $15,48 \mathrm{a}$ & $22,63 \mathrm{a}$ & $20,27 \mathrm{a}$ \\
3)etanol & $47,23 \mathrm{a}$ & $45,22 \mathrm{a}$ & $8,12 \mathrm{a}$ & $10,29 \mathrm{~b}$ & $21,37 \mathrm{a}$ & $21,82 \mathrm{a}$ \\
4) $\mathrm{CaCl}_{2}$ & $47,82 \mathrm{a}$ & $42,55 \mathrm{a}$ & $4,16 \mathrm{a}$ & $14,23 \mathrm{ab}$ & $25,17 \mathrm{a}$ & $14,91 \mathrm{~b}$ \\
\hline $\mathrm{CV}(\%)$ & 5,5 & 6,4 & 52,4 & 25,6 & 19,11 & 14,27 \\
\hline
\end{tabular}

${ }^{(1)}$ Média de 10 repetições. Médias seguidas de mesma letra na coluna não diferem significativamente entre si (Tukey, $\left.\mathrm{p} \leq 0,05\right)$.

TABELA 2- Coloração da casca ( $\mathrm{L}^{*}=$ luminosidade; $+\mathrm{a}^{*}=$ vermelho; $-\mathrm{a}^{*}=$ verde; $+\mathrm{b} *=$ amarelo; $-\mathrm{b}^{*}=$ azul) de ameixas cv. Reubennel, submetidas a tratamentos pós-colheita, armazenadas a $0^{\circ} \mathrm{C}$ por 17 e 33 dias, mais 4 dias sob condições ambiente $\left(20^{\circ} \mathrm{C}\right)$, com ou sem climatização.

\begin{tabular}{|c|c|c|c|c|c|c|}
\hline \multirow{2}{*}{ Tratamentos } & \multicolumn{2}{|c|}{$\mathrm{L}^{*}$} & \multicolumn{2}{|c|}{$\mathrm{a}^{*}$} & \multicolumn{2}{|c|}{$\mathrm{b}^{*}$} \\
\hline & $17+4$ dias & $33+4$ dias & $17+4$ dias & $33+4$ dias & $17+4$ dias & $33+4$ dias \\
\hline 1)testemunha & $41,59 \mathrm{aA}^{(1)}$ & 39,98 aA & $14,86 \mathrm{aA}$ & $15,49 \mathrm{aA}$ & $10,31 \mathrm{aA}$ & $6,84 \mathrm{aA}$ \\
\hline 2)ECOLIFE® & $41,68 \mathrm{aA}$ & $35,76 \mathrm{cA}$ & $13,63 \mathrm{aA}$ & $13,98 \mathrm{aA}$ & 8,29 aA & $5,43 \mathrm{abA}$ \\
\hline 3)etanol & $41,73 \mathrm{aA}$ & $37,20 \mathrm{bcA}$ & $13,66 \mathrm{aA}$ & $10,51 \mathrm{bA}$ & $7,04 \mathrm{aA}$ & $3,09 \mathrm{cA}$ \\
\hline 4) $\mathrm{CaCl}_{2}$ & $41,00 \mathrm{aA}$ & $37,84 \mathrm{bA}$ & $14,78 \mathrm{aA}$ & $10,77 \mathrm{bA}$ & $8,54 \mathrm{aA}$ & $3,28 \mathrm{bcA}$ \\
\hline Média & $41,50 \mathrm{~A}$ & $37,69 \mathrm{~A}$ & $14,23 \mathrm{~A}$ & $12,69 \mathrm{~A}$ & $8,54 \mathrm{~A}$ & $4,66 \mathrm{~A}$ \\
\hline 1E)testemunha + etileno & $35,36 \mathrm{bB}$ & $35,07 \mathrm{aB}$ & $10,79 \mathrm{aA}$ & $6,87 \mathrm{aB}$ & $3,70 \mathrm{aA}$ & $0,44 \mathrm{aB}$ \\
\hline 2E)ECOLIFE® + etileno & $36,67 \mathrm{aB}$ & $36,21 \mathrm{aA}$ & $9,84 \mathrm{aA}$ & $6,21 \mathrm{aB}$ & $2,85 \mathrm{aA}$ & $0,64 \mathrm{aB}$ \\
\hline $3 \mathrm{E})$ etanol + etileno & $37,11 \mathrm{aB}$ & $36,42 \mathrm{aA}$ & $9,03 \mathrm{aA}$ & $7,07 \mathrm{aB}$ & $2,77 \mathrm{aA}$ & $0,99 \mathrm{aB}$ \\
\hline $4 \mathrm{E}) \mathrm{CaCl}_{2}+$ etileno & $37,27 \mathrm{aB}$ & $35,53 \mathrm{aB}$ & $9,40 \mathrm{aA}$ & $7,27 \mathrm{aB}$ & 3,11 aA & $1,04 \mathrm{aB}$ \\
\hline Média & $36,60 \mathrm{~B}$ & $35,80 \quad \mathrm{~B}$ & $9,76 \mathrm{~B}$ & $6,85 \mathrm{~B}$ & $3,11 \mathrm{~B}$ & $0,78 \mathrm{~B}$ \\
\hline Fator A (trat.) & ns & $*$ & ns & $*$ & $* *$ & $*$ \\
\hline Fator B (etileno) & $* *$ & $* *$ & $* *$ & $* *$ & $* *$ & $* *$ \\
\hline Fator A x B & $*$ & ** & ns & $* *$ & ns & $* *$ \\
\hline $\mathrm{CV}(\%)$ & 3,6 & 4,0 & 15,2 & 25,6 & 30,2 & 67,3 \\
\hline
\end{tabular}

${ }^{(1)}$ Média de 10 repetições. Médias seguidas de mesma letra na coluna não diferem significativamente entre si (Tukey, $\left.\mathrm{p} \leq 0,05\right)$. Letras minúsculas representam as diferenças entre tratamentos na coluna, comparando de 1 a 4 , e de $1 \mathrm{E}$ a $4 \mathrm{E}$, respectivamente. Letras maiúsculas representam diferenças da análise fatorial dos tratamentos, comparando cada tratamento com e sem etileno (ex. 1x1E). 
TABELA 3- Firmeza da polpa (N), sólidos solúveis ( ${ }^{\circ}$ Brix) e acidez titulável ( $\mathrm{g}$ ác. cítrico $\left.100 \mathrm{~g}^{-1}\right)$ de ameixas cv. Reubennel, submetidas a tratamentos pós-colheita, armazenadas a $0^{\circ} \mathrm{C}$ por 17 e 33 dias.

\begin{tabular}{lcccccc}
\hline \multirow{2}{*}{ Tratamentos } & \multicolumn{2}{c}{ Firmeza $(\mathrm{N})$} & \multicolumn{2}{c}{$\mathrm{SS}\left({ }^{\circ} \mathrm{Brix}\right)$} & \multicolumn{2}{c}{$\mathrm{AT}\left(\mathrm{g} 100 \mathrm{~g}^{-1}\right)$} \\
\cline { 2 - 7 } & 17 dias & 33 dias & 17 dias & 33 dias & 17 dias & 33 dias \\
1)testemunha & $8,99 \mathrm{a}^{(1)}$ & $8,32 \mathrm{a}$ & $11,4 \mathrm{a}$ & $11,2 \mathrm{a}$ & $1,27 \mathrm{a}$ & $1,13 \mathrm{a}$ \\
2)ECOLIFE® & $8,17 \mathrm{a}$ & $7,18 \mathrm{ab}$ & $11,3 \mathrm{a}$ & $11,9 \mathrm{a}$ & $1,22 \mathrm{a}$ & $1,12 \mathrm{a}$ \\
$3)$ etanol & $8,55 \mathrm{a}$ & $7,41 \mathrm{ab}$ & $11,1 \mathrm{a}$ & $12,0 \mathrm{a}$ & $1,29 \mathrm{a}$ & $1,10 \mathrm{a}$ \\
$4) \mathrm{CaCl}_{2}$ & $8,59 \mathrm{a}$ & $5,82 \mathrm{~b}$ & $10,1 \mathrm{~b}$ & $11,8 \mathrm{a}$ & $1,26 \mathrm{a}$ & $1,25 \mathrm{a}$ \\
$\mathrm{CV}(\%)$ & 24,0 & 33,6 & 5,2 & 5,5 & $1,27 \mathrm{a}$ & $1,13 \mathrm{a}$ \\
\hline
\end{tabular}

${ }^{(1)}$ Média de 10 repetições. Médias seguidas de mesma letra na coluna não diferem significativamente entre si (Tukey, $\mathrm{p} \leq 0,05$ ).

TABELA 4- Firmeza da polpa (N), sólidos solúveis ( ${ }^{\circ}$ Brix) e acidez titulável ( $\mathrm{g}$ ác. cítrico $\left.100 \mathrm{~g}^{-1}\right)$ de ameixas cv. Reubennel, submetidas a tratamentos pós-colheita, armazenadas a $0^{\circ} \mathrm{C}$ por 17 e 33 dias, mais 4 dias sob condições ambiente $\left(20^{\circ} \mathrm{C}\right)$, com ou sem climatização.

\begin{tabular}{|c|c|c|c|c|c|c|}
\hline \multirow{2}{*}{ Tratamentos } & \multicolumn{2}{|c|}{ Firmeza $(\mathrm{N})$} & \multicolumn{2}{|c|}{$\mathrm{SS}\left({ }^{\circ}\right.$ Brix $)$} & \multicolumn{2}{|c|}{ AT $\left(g_{\left.100 g^{-1}\right)}\right.$} \\
\hline & $17+4$ dias & $33+4$ dias & $17+4$ dias & $33+4$ dias & $17+4$ dias & $33+4$ dias \\
\hline 1)testemunha & $7,50 \mathrm{aA}$ & $7,26 \mathrm{aA}$ & 11,4 bcA & $11,0 \mathrm{aA}$ & $1,29 \mathrm{aA}$ & $0,99 \mathrm{aA}$ \\
\hline 2)ECOLIFE® & $5,77 \mathrm{bA}$ & $4,75 \mathrm{bA}$ & $11,8 \mathrm{abA}$ & $11,9 \mathrm{aA}$ & $1,21 \mathrm{aA}$ & $1,04 \mathrm{aA}$ \\
\hline 3)etanol & $6,08 \mathrm{bA}$ & $4,04 \mathrm{bcA}$ & $12,0 \mathrm{aA}$ & $12,6 \mathrm{aA}$ & $1,20 \mathrm{aA}$ & $0,96 \mathrm{aA}$ \\
\hline 4) $\mathrm{CaCl}_{2}$ & $5,69 \mathrm{bA}$ & $2,73 \mathrm{cA}$ & $11,2 \mathrm{cA}$ & $11,9 \mathrm{aA}$ & $1,25 \mathrm{aA}$ & $0,92 \mathrm{aA}$ \\
\hline Média & $6,26 \mathrm{~A}$ & $4,69 \mathrm{~A}$ & $11,6 \mathrm{~A}$ & $11,8 \mathrm{~A}$ & $1,24 \mathrm{~A}$ & $0,98 \mathrm{~A}$ \\
\hline 1E)testemunha + etileno & $0,89 \mathrm{aB}$ & $1,01 \mathrm{aB}$ & $11,5 \mathrm{bA}$ & $11,2 \mathrm{aA}$ & $1,01 \mathrm{aA}$ & $0,84 \mathrm{aA}$ \\
\hline 2E)ECOLIFE $\AA+$ etileno & $0,87 \mathrm{aB}$ & $1,47 \mathrm{aB}$ & $11,1 \mathrm{bB}$ & $12,6 \mathrm{aA}$ & $0,97 \mathrm{aA}$ & $0,85 \mathrm{aA}$ \\
\hline 3E)etanol + etileno & $0,92 \mathrm{aB}$ & $1,03 \mathrm{aB}$ & $12,1 \mathrm{aA}$ & $12,7 \mathrm{aA}$ & $0,93 \mathrm{aA}$ & $0,86 \mathrm{aA}$ \\
\hline 4E) $\mathrm{CaCl}_{2}+$ etileno & $0,90 \mathrm{aB}$ & $1,64 \mathrm{aB}$ & $11,1 \mathrm{bA}$ & $11,7 \mathrm{aA}$ & $0,96 \mathrm{aA}$ & $0,85 \mathrm{aA}$ \\
\hline Média & $0,90 \mathrm{~B}$ & $1,29 \mathrm{~B}$ & $11,4 \mathrm{~A}$ & $12,0 \mathrm{~A}$ & $0,96 \mathrm{~B}$ & $0,85 \mathrm{~B}$ \\
\hline Fator A (trat.) & $*$ & $* *$ & $* *$ & $* *$ & $*$ & $\mathrm{~ns}$ \\
\hline Fator B (etileno) & $* *$ & $* *$ & ns & ns & $* *$ & $* *$ \\
\hline Fator A x B & $*$ & $* *$ & $*$ & ns & ns & ns \\
\hline $\mathrm{CV}(\%)$ & 43,0 & 57,1 & 4,2 & 6,5 & 8,1 & 11,9 \\
\hline
\end{tabular}

${ }^{(1)}$ Média de 10 repetições. Médias seguidas de mesma letra na coluna não diferem significativamente entre si (Tukey, $\left.\mathrm{p} \leq 0,05\right)$. Letras minúsculas representam as diferenças entre tratamentos na coluna, comparando de 1 a 4, e de 1E a 4E, respectivamente. Letras maiúsculas representam diferenças da análise fatorial dos tratamentos, comparando cada tratamento com e sem etileno (ex. 1x1E).

\section{CONCLUSÃO}

O tratamento pós-colheita das ameixas com vapor de etanol reduz a população microbiana, bem como proporciona incremento dos sólidos solúveis. As ameixas 'Reubennel' podem ser armazenadas por até 33 dias sob refrigeração $\left(0^{\circ} \mathrm{C} / 95 \% \mathrm{UR}\right)$, seguindose a climatização com etileno e, imediatamente, comercializadas.

\section{AGRADECIMENTOS}

Ao Sr. Renato Mindlin Loeb, que com este trabalho obteve uma consultoria com apoio do SEBRAE-SP, através do Programa SEBRAETEC de Consultoria Tecnológica, em parceria com o ITAL. 


\section{REFERÊNCIAS}

ABREU, F.M.; LOURENÇO, S.A.; BASSETTO, E.; GONÇALVES, F.P.; MARTINS, M.C.; AMORIM, L. Efeito de sanificantes no controle pós-colheita da podridão parda (Monilinia fructicola) e da podridão mole (Rhizopus stolonifer) em pêssegos. Summa Phytopathologica, Botucatu, v.34, n.1, p.86-88, 2008 .

ARGENTA, L.C.; KRAMMES, J.G.; MEGGUER, C.A.; AMARANTE, C.V.T.; MATTHEIS, J. Ripening and quality of 'Laetitia' plums following harvest and cold storage as affected by inhibition of ethylene action. Pesquisa Agropecuária Brasileira, Brasília, v.38, n.10, p.1139-1148, 2003.

BRACKMANN, A.; BENEDETTI, M.; HUNSCHE, M.; SESTARI, I. Armazenamento de ameixas cv. Reubennel e Pluma 7 sob diferentes temperaturas, em atmosfera controlada e refrigerada. Revista Brasileira de Agrociência, Pelotas, v.11, n.1, p. 85-89, 2005.

BRACKMANN, A.; STEFFENS, C.A.; MELLO, A.M. de. Efeito do pré-resfriamento e temperatura de armazenamento na qualidade de ameixas, cv. Pluma 7 e Reubennel. Revista Brasileira de Agrociência, Pelotas, v.7, n.1, p.18-21, 2001.

BRASIL. Ministério da Saúde. Agência Nacional de Vigilância Sanitária - ANVISA. Resolução RDC n 12, de 02 de janeiro de 2001. Aprova o Regulamento Técnico sobre padrões microbiológicos para alimentos. Diário Oficial da República Federativa do Brasil, Brasília, DF, 10 jan. 2001.

CANDAN, A.P.; GRAELL, J.; LARRIGAUDIERE, C. Postharvest quality and chilling of plums: benefits of 1-methylcyclopropene. Spanish Journal of Agricultural Research, Madri, v.9, n.2, p.554-564, 2011.

CRISOSTO, C.H.; KADER, A.A. Plum and fresh prune postharvest quality maintenance guidelies. 2000. v.10, 8p. Disponível em: http://www.uckac. edu/postharv/PDF\%20files/Guidelines/plum.pdf. Acesso em: 10 jan. 2013.

DOWNES, F.P.; ITO, K. (Ed.). Compendium of methods for the microbiological examination of foods. $4^{\text {th }}$ ed. Washington: American Public Health Association, 2001. p.357-380.
ERKAN, M.; ESKI, H. Combined treatment of modified atmosphere packaging and 1-methylcyclopropene improves postharvest quality of Japanese plums. Turkish Journal of Agriculture Forestry, Ankara, v.36, p.563-575, 2012.

FIORAVANÇO, J.C.; PAIVA, M.C.; BIZZANI, E. Ethephon na antecipação da colheita e qualidade da ameixa cv. Reubennel. Scientia Agrícola, Piracicaba, v.8, n.2, p. 193-197, 2007.

GONÇALVES, F. P. Quantificação de danos e controle pós-colheita da podridão parda (Monilinia fructicola) e podridão mole (Rhizopus stolonifer) em frutos de ameixa e nectarina. 2005.73f. Dissertação (Mestrado em Fitopatologia)Escola Superior de Agricultura "Luiz de Queiroz", Universidade de São Paulo, Piracicaba, 2005.

KLUGE, R.A.; HOFFMANN, A.; NACHTIGAL, J.C.; BILHALVA, A.B. Qualidade de ameixas (Prunus salicina, Lindl.) 'Reubennel' após armazenamento refrigerado. Scientia Agrícola, Piracicaba, v.52, n.3, p.476-481, 1995.

KLUGE, R.A.; JACOMINO, A.P.; ALVES, R.M.; CASTRO, P.R.C.; AGUILA, J.S. Efeito do 1-metilciclopropeno em ameixa e nectarina. Revista Iberoamericana de Tecnologia Postcosecha, Hermosillo, v.9, n.1, p.26-33, 2008.

LICHTER, A.; GABLER, F.M.; SMILANICK, J.L. Control os spoilage in table grapres. Stewart Postharvest Review, Quebec, v.6, n.1, p.1-10, 2006.

MALGARIM, M. B.; CANTILLANO, R. F. F.; TREPTOW, R. O.; SOUZA, E.L. COUTINHO, E.F. Modificação da atmosfera na qualidade pós-colheita de ameixas cv. Reubennel. Revista Brasileira de Fruticultura, Jaboticabal, v.27, n.3, p.373-378, 2005.

MALGARIM, M. B.; CANTILLANO, R. F. F.; TREPTOW, R. O; SOUZA, E. L. Estádio de maturação e variação da temperatura na qualidade pós-colheita de ameixas cv. Reubennel. Revista Brasileira de Agrociência, Pelotas, v.13, n.1, p. 61-67, 2007.

MORAES, M.A.C. Métodos para avaliação sensorial dos alimentos. 6.ed. Campinas: UNICAMP, 1988. 93p. 
NASCIMENTO, L. M.; YOTSUYANAGI, K.; COUTINHO, J. R. Efeito do cloreto de cálcio bihidratado no armazenamento refrigerado de ameixas (Prunus sp.). Revista Brasileira de Fruticultura, Jaboticabal, v.15, n.1, p.1-8, 1993.

PALOU, L.; CRISOSTO, C.H.; GARNER, D.; BASINAL, L.M. Effect of continous exposure to exogenous ethylene during cold storage on postharvest decay development and quality attributes of stone fruits and table grapes. Postharvest Biology and Technology, Amsterdam, v.27, p.243-254, 2003.

PESIS, E. The role of the anaerobic metabolites, acetaldehyde and ethanol, in fruit ripening, enhancement of fruit quality and fruit deterioration. Postharvest Biology and Technology, Amsterdam, v.37, p.1-19, 2005.
RITENOUR, M.A.; MANGRICH, M.E.; BEAULIEU, J.C.; RAB, A.; SALTVEIT, M.E. Ethanol effects on the ripening of climacteric fruit. Postharvest Biology and Technology, Amsterdam, v.12, p.35-42, 1997.

SIDDIQUI, S.; KOVACS, E; BECZNER, J.; GOYAL, R.K.; GARG, F.C. Effect of ethanol, acetic acid and hot water vapours on the shelf-life of guava (Psidium guajava L.). Acta Alimentaria, Budapest, v.34, n.1, p. 49-57, 2005.

STEFFENS, C. A.; AMARANTE, C. V. T.; ALVES, E. O.; TANAKA, H.; BRACKMANN, A.; BOTH, V. Armazenamento de ameixas 'Laetitia' em atmosfera modificada. Ciência Rural, Santa Maria, v.39, n.9, p.2439-2444, 2009. 